\title{
Developing a conceptual model for the application of patient and public involvement in the healthcare system in Iran
}

\author{
Mohammad Azmal $^{1}$, Ali Akbari Sari ${ }^{2}$, Abbas Rahimi Foroushani $^{3}$, Batoul Ahmadi ${ }^{4,5}$
}

${ }^{1}$ Ph.D. Candidate of Health Services Management, Department of Health Management and Economics, School of Public Health, Tehran University of Medical Sciences, Tehran, Iran

${ }^{2}$ Ph.D. of Health Policy and Management, Associate Professor, Department of Health Management and Economics, School of Public Health, Tehran University of Medical Sciences, Tehran, Iran

${ }^{3} \mathrm{Ph} . \mathrm{D}$. of Biostatistics, Professor, Department of Epidemiology and Biostatistics, School of Public Health, Tehran University of Medical Sciences, Tehran, Iran

${ }^{4}$ Ph.D. of Health Services Management, Associate Professor, Department of Health Management and Economics, School of Public Health, Tehran University of Medical Sciences, Tehran, Iran

${ }^{5}$ Women's Health Scientific Group, Iranian Academy of Medical Sciences, Tehran, Iran

\section{Type of article: Original}

\begin{abstract}
Introduction: Patient and public involvement is engaging patients, providers, community representatives, and the public in healthcare planning and decision-making. The purpose of this study was to develop a model for the application of patient and public involvement in decision making in the Iranian healthcare system.

Methods: A mixed qualitative-quantitative approach was used to develop a conceptual model. Thirty three key informants were purposely recruited in the qualitative stage, and 420 people (patients and their companions) were included in a protocol study that was implemented in five steps: 1) Identifying antecedents, consequences, and variables associated with the patient and the publics' involvement in healthcare decision making through a comprehensive literature review; 2) Determining the main variables in the context of Iran's health system using conceptual framework analysis; 3) Prioritizing and weighting variables by Shannon entropy; 4) designing and validating a tool for patient and public involvement in healthcare decision making; and 5) Providing a conceptual model of patient and the public involvement in planning and developing healthcare using structural equation modeling. We used various software programs, including SPSS (17), Max QDA (10), EXCEL, and LISREL. Content analysis, Shannon entropy, and descriptive and analytic statistics were used to analyze the data.

Results: In this study, seven antecedents variable, five dimensions of involvement, and six consequences were identified. These variables were used to design a valid tool. A logical model was derived that explained the logical relationships between antecedent and consequent variables and the dimensions of patient and public involvement as well.

Conclusion: Given the specific context of the political, social, and innovative environments in Iran, it was necessary to design a model that would be compatible with these features. It can improve the quality of care and promote the patient and the public satisfaction with healthcare and legitimate the representative of people they served for. This model can provide a practical guide for managers and policy makers to involve people in making the decisions that influence their lives.

Keywords: Patient and public involvement, Conceptual model, Healthcare system, Iran
\end{abstract}

\section{Corresponding author:}

Associate Professor Dr. Batoul Ahmadi, Department of Health Management and Economics, School of Public Health, Tehran University of Medical Sciences, Tehran, Iran.

Tel: +98.2188951391, Fax:+98.2188989129, Email: ahmadiba@tums.ac.ir

Received: November 01, 2015, Accepted: February 19, 2016, Published: June 2016

iThenticate screening: February 19, 2016, English editing: April 16, 2016, Quality control: June 02, 2016

(c) 2016 The Authors. This is an open access article under the terms of the Creative Commons Attribution-NonCommercialNoDerivs License, which permits use and distribution in any medium, provided the original work is properly cited, the use is non-commercial and no modifications or adaptations are made. 


\section{Introduction \\ 1.1. Background}

Involving the public in decision making can be understood as a means of restoring confidence, improving accountability, and ensuring cost-effective decisions in the healthcare system (1). Citizens' participation in the planning, delivery, and evaluation of health services was conceptualized for the first time by the World Health Organization (WHO) during the Alma-Ata Conference in 1978 (2). Community-based involvement is the essential base for the health policymaking process in the UK, Canada, and Australia $(3,4)$. Different countries also have experienced projects, such as public consultation, to conduct their priorities (5). The Iranian Ministry of Health and Medical Education (MOHME) delivers healthcare through its health services network. The MOHME is responsible for policy development, supervision, regulation, planning, and designing the healthcare system, including medical insurance, medical education, and research (6). The MOHME delegates some of its tasks related to policy implementation of healthcare programs in addition to assigning medical education to the Universities of Medical Sciences and Health Services (UMSHS) at the province level (7). UMSHS provides healthcare services to the population through a district health network and district hospitals at the urban level and by health houses in the rural areas (6). In 2002, MOHME announced the patient rights charter to support the patients (8). The charter includes five areas, i.e., 1) the right to receive good services, 2) the right to receive adequate information, 3) the right to freely choose and make decisions about health care, 4) respect for privacy, and 5) confidentiality of patient and compliance with the principle of access to an effective complaints system (9). But the "patient and public involvement" concept entered the Iranian health system context with the implementation of clinical governance (CG) as an accepted model for improving the quality of hospital care, and it became a task for all hospitals across the country (10). In November 2009, the Ministry of Health introduced the Seven Pillars model of clinical governance for all hospitals nationwide to prepare necessary infrastructure for the implementation of the clinical governance mandates in accordance with the model (11). The CG office was established to monitor the programs and set some priorities to establish a supportive culture for quality improvement (12) However, it seems that this axis of program was defined very well among the other topics, and it was not adequately comprehensive and was limited to the tenets of the previous program, such as investigating complaints and discharge against medical advice (DAMA) programs.

\subsection{Statement of the problem and study logic}

The patient and public involvement (PPI) is the active involvement of patients, care providers, community representatives, community groups, and public in such a way that services are planned, delivered, and evaluated (13). In fact, it is the process by which concerns, needs, and values of the society become engaged in decisionmaking (14). Patient involvement can be considered useful for healthcare system policy and planning activities (15). Their participation also can ensure informed decision on the distribution and delivery of healthcare (16). Evidence suggests that participation can provide the opportunity to achieve better quality of care, greater cost effectiveness, and improvements in the population's health (17). Patient, the public and other stakeholders' education can be an effective factor in implementing PPI successfully, because it will create a culture of participation, develop a legal base for PPI, allow careful selection of participants, and ensure that the methods used are transparent (18). Despite the growing body of literature on PPI, this concept has remained poorly defined, and numerous questions remain about applying the appropriate approach (19). There is no single model to cover the fundamental concept of public involvement comprehensively (20). However, it is essential to formulate the principles of involvement that are generalizable and applicable across national and political environments. Standardization of the involvement methods also should be compatible with and sensitive to local conditions (21). In this project, our aim was to develop a logical conceptual model of PPI in Iranian healthcare decision making with respect to the specific political, social, and cultural context of Iran. We tried to anticipate the prerequisites of effective implementation of PPI and their possible consequences.

\subsection{Objectives}

The specific objectives of this study were: 1) to identify antecedents, dimensions, and consequent variables of PPI by reviewing the literature and conducting semi-structured interviews with key informants, 2) to prioritize and weigh the antecedent, dimensions, and essential variables using Shannon entropy, 3) to develop and validate tools to assess PPI's involvement in decision making concerning Iranian healthcare, and 4) to use Structural Equation Modeling (SEM) to develop a conceptual model of PPI for the application of patient and the public involvement in the healthcare system in Iran. 


\section{Material and Methods}

The current protocol study was conducted using a mixed qualitative-quantitative approach to collect data and analyze results. The statistical methods were used to assess the power relationships between the variables. These relationships were presented in structured diagrams using structural equation modeling (SEM). This study consisted of the following five steps.

\subsection{Step one: literature review}

The keywords included patient and the public involvement, decision-making, and other similar words that were determined in consultation with a librarian. Inclusion and exclusion criteria for the screening of applications were determined as follows. First, articles must be included that address the patients, the public, or their representatives. Second, the articles should encompass the process of involvement (e.g., communication, consultation, participation, and engagement). Third, it should be referred to a decision-making or policymaking process in the healthcare system, and the papers must be published in Farsi and English between 2000 and 2015. Based on the exclusion criteria, studies on patient involvement in decision-making at the individual level were deleted. Similarly, articles in the field of PPI in healthcare research and developing clinical guidelines were the exclusion criteria for this study. At the end of this step, the antecedent and the consequence variables and dimensions of PPI in healthcare decision making were identified and classified. The purpose of this step was to identify and understand the range of factors that affect PPI in healthcare decisions. This was achieved through an extensive review of the literature on PPI, and the antecedent and consequence variables and dimensions of PPI also were extracted. In this step, the relevant articles about PPI were extracted using the proper keywords in various databases, such as Pubmed, ISI Web of Science, Scopus, PsycNET, EMBASE, ScienceDirect, and Google Scholar. The Persian databases, including Magiran, Scientific Information Database (SID), Irandoc, and Civilica, were used as well. An obvious search strategy was developed to identify related papers about PPI. Keywords included patient and the public involvement, decision-making, and other similar words that were identified in consultation with a librarian. Inclusion and exclusion criteria for the screening of applications were determined as follows: First, the articles must include patients, the public, or their representatives. Second, the articles should encompass the process of involvement (e.g., communication, consultation, participation, and engagement). Third, it should be related to decision-making or policymaking in the healthcare system, and, fourth, the papers must have been published in Farsi and English between 2000 and 2015. Based on the exclusion criteria, studies on patient involvement in decision-making at the individual level were deleted. Similarly, articles in the field of PPI in healthcare research and developing clinical guidelines were the exclusion criteria for this study. The information gathered from studies were retrieved based on information sources and synthesis them. At the end of this step, the antecedent, consequence variables, and dimensions of PPI in healthcare decision making were identified and classified.

\subsection{Step two: qualitative study}

As we noted earlier, there is a close association between involvement methods and the context in which PPI occurs (20). In this regard, more research will be required to determine which method is more effective in various contexts. The context may include the political context (for example, the relationships between the government and the public), the social context (including the characteristics of the population, geography, political participation, social capital), the innovation context (considering academic expertise), the organizational context (e.g., limitations, amount and level of commitment to the project, and the attitudes of the organization's key employees), and the decision context (decision making procedure, the different actors' responsibilities, different issues, the decisionmaking process and schedules). In this regard, the components of PPI in decision-making it should be assessed in the context of the Iranian healthcare system, which has special characteristics. Particularly, this community has cultural, religious, and political contexts that are unique. So, it is better to identify the variables that represent the characteristics of the context. Therefore, the qualitative method in semi-structured interviews (conceptual model approach) was used to assess the Iranian context status. The key informants included relevant policy makers and top managers in MOHME, at the University of Medical Sciences, among the representatives from the medical professions and health institutions, patients and their companions, and researchers and experts from the field of PPI in Iran. The key informants were selected using the purposive and snowball sampling strategy with in an effort to identify qualified experts who have then desired theoretical and practical perspectives of PPI in healthcare decisionmaking. These people mainly fit into two groups i.e., 1) decision-makers or experts and 2) healthcare managers, scholars, and researchers in the PPI field. Key informants were eligible to participate in the study if they had more than five years' experience in decision making and policymaking in the healthcare system. Potential interviewees via email or by phone were invited to participate and be interviewed. An "interview guide" was developed to provide the structure of the interviews and to ensure that the focus of the interviews was on obtaining the views and 
experiences of key informants. Sampling continued until data saturation was attained so that the participants were not providing any new information. After each interview, all of its content was recorded with the participants' permission. Then, the interviews were transcribed, and relevant code was extracted using Max QDA software version 10. To ensure the accuracy and reliability of data (Credibility, Dependability, Transferability and Conformability), different methods were used in the study, such as peer checking, member checking, and expert checking.

\subsection{Step three: prioritizing the factors}

In this step, we prioritized the components that were obtained from the previous steps. In this way, only the most important and highest priority components, including the antecedents and consequence variables of PPI, were determined for use in the next step. To analyze the data in this step, weighting and prioritizing variables were done using the Shannon entropy technique. In this regard, the expert opinion before (qualitative research) was asked. A data collection form was used in weighting the variables. Three important criteria were entered in the abovementioned form, i.e., the necessity, importance, and relevance of these variables with PPI in healthcare decisionmaking, and, then, the participants were asked to provide weighting variables and indicators. When the decisionmaking has been specified completely by a matrix, the entropy method can be used to measure the weights of the variables (22). All operations described in this section (weighting and ranking) were conducted using Excel 2010 in the Microsoft Office Software.

\subsection{Step four: design and validation of the questionnaire}

As previously mentioned, the antecedent and consequence variables were identified during the literature review and qualitative steps. In this step, a primary questionnaire was designed using the results of the previous steps to assess the PPI. In this way, questions to be answered on a five-point Likert scale were designed to extract the dimensions. After providing the initial draft of the questionnaire, we attempted to standardize it. The purpose of standardization at this stage of the study was to assess the reliability, face validity, content validity, and construct validity. To assess the face validity, the experts' judgments were used. Content validity was measured using the Lawshe method. Construct validity also was measured by confirmatory factor analysis (CFA) and applying LISREL software. To assess the reliability of the questionnaire, Cronbach's alpha was used. The questionnaire was distributed to the research community or stakeholders at the operational level, based on required the sample size. These stakeholders were assigned to one of two separate groups, i.e., 1) individuals and their representatives of the supply side, including the managers and decision makers in healthcare institutions (hospitals), representatives of healthcare professions (doctors in all specialties and disciplines, nursing groups and other groups associated with different groups of patients) and 2) individuals and their representatives of the demand side, such as patients and their family members, representatives of organizations, and Non-governmental organizations NGOs ,Consumer advocacy organizations and other organizations, such as the association of specific diseases. In addition, in order to keep an appropriate balance between the supply and demand sides, the samples were selected from the six selected cities in Iran. Sampling from patients and their relatives were based on sequential sampling, and sampling in supply groups was conducted according a quota that was proportional to their population. The individuals and groups from all of the selected cities based on the patterns of rationalization in hospitalization and specialized medical care for Iran, including the cities of Tehran, Tabriz, Shiraz, Mashhad, Ahwaz, and Kermanshah, were enrolled in this study so the sample would be representative of the general population and the results could be generalized to the entire country. We distributed 420 questionnaires among selected samples in general hospitals of the cities mentioned above. Data were entered in SPSS version 16. Descriptive statistics were used to summarize demographic data and central and variation parameters, such as mean and standard deviation, in tables. Also, appropriate statistical tests, including Independent t-test and ANOVA, were used to verify the significance level of the PPI dimensions in terms of demographic factors and Pearson's correlation coefficient to measure the correlation coefficient between variables.

\subsection{Step five: proposing a suitable model}

Since this step was a complete phase, the data that were collected in the previous step were used. In this step, new data were not collected, and the data collected from the questionnaire were analyzed using LISREL software for modeling. We presented a conceptual model of PPI using structural equation modeling (SEM). It will be used for some complementary tests, including Goodness of Fit Index (GFI), Adjusted Goodness of Fit Index (AGFI), Root Mean Square Error of Approximation (RMSEA), the square root of the mean square error or Chi, Akaike information criterion (AIC), and Non-normed Fit Index (NNFI) to assess the appropriateness of the model. Finally, if the initial fit indexes were not appropriate, the basic model was modified based on the error covariance between 
variables, and the relationships between them were adjusted as the software suggested, thereby improving the fit index.

\section{Results}

In the first step (literature review) 11,760 papers were identified from main databases and other sources. After removing irrelevant and duplicate titles, 419 papers were selected for the quality appraisal, and, finally, 59 papers were deemed to be eligible for inclusion. After access to the full text of selected studies, data were extracted based on the research question in this regards, eight antecedent variables (leadership, legitimacy, representativeness, public empowerment, participation culture, the flow of information and communication, involvement process, and effective structure), and 25 subtitles also were extracted from the literature. In addition, five levels of public involvement were selected from the IAP2 (International Association for Public Participation) were used. These levels consisted of inform, consult, involve, collaborate, and empower.

Consequently, seven variables were extracted from the selected studies and 25 subtitles, i.e., 1) effective decision making, 2) intermediate consequence, such as efficiency, productivity, trust, and justice, 3) constructive information exchange, 4) effective communication, 5) good health, 6) personal and public development, and 7) patient and the public outcomes. In the qualitative steps, we focused on the context of Iranian healthcare. So six main themes and 28 sub-themes were identified after conducting semi-structured interviews with 33 key informants. The main themes as antecedent variables were 1) including requirements for participation, 2) culture and ethics, 3) education and empowerment, 4) democracy and representation, 5) appropriate structure, and 5) the participation process. Also, seven consequences were identified, including 1) prevention of negative consequences, 2) health and satisfaction, 3) effectiveness and efficiency, 4) strengthen the voice of the customer, 5) trust and social capital, 6) better decisions, and 7) development of programs and services. Sixty-three antecedent variables and 35 consequent variables were identified from earlier steps, and they were prioritized and weighted using Shannon entropy.

A checklist was developed and sent to 15 key informants who collaborated in the qualitative step, and they determined the variables based on their importance, necessity, and relation to PPI. At the end of this stage, 42 antecedent variables and 26 consequent variables were selected to enter in the next step. A primary questionnaire was designed in step four based on the variables that were identified in steps 1-3. This questionnaire consisted of 42 items evaluated with a 5-point Likert scale (strongly disagree to strongly agree) to assess the PPI antecedent in seven categories and 25 items for the PPI dimension in five categories and 26 items for the consequence of PPI in six categories. We also had to validate this tool. For this, the face validity was approved by expert opinion, and content validity also was acquired by calculating Content validity Ratio (CVR). The final questionnaire that was developed included 35, 25, and 22 items for antecedent, dimension, and consequent of PPI, respectively. Test and re-test were used to assess the reliability of the questionnaire for which CCI was 0.875 , indicating strong reliability. The questionnaire was distributed to the targeted samples, including 210 people on the demand side (patients and their companions) and 210 people from the supply side (physicians, nurses, and other staff). At the time this manuscript was submitted, steps one through four of the study were complete, and the last step (confirming the model) was underway. In this regard, we are going to determine the relations between antecedent, dimension, and consequent variables using structural equation modeling (SEM). These relations are shown in Figure 1. Figure 1 shows the proposed conceptual model of PPI in healthcare decision making. This model can be used to calculate the effect of antecedent and consequence variables, the dimensions of PPI, and its coefficients. 


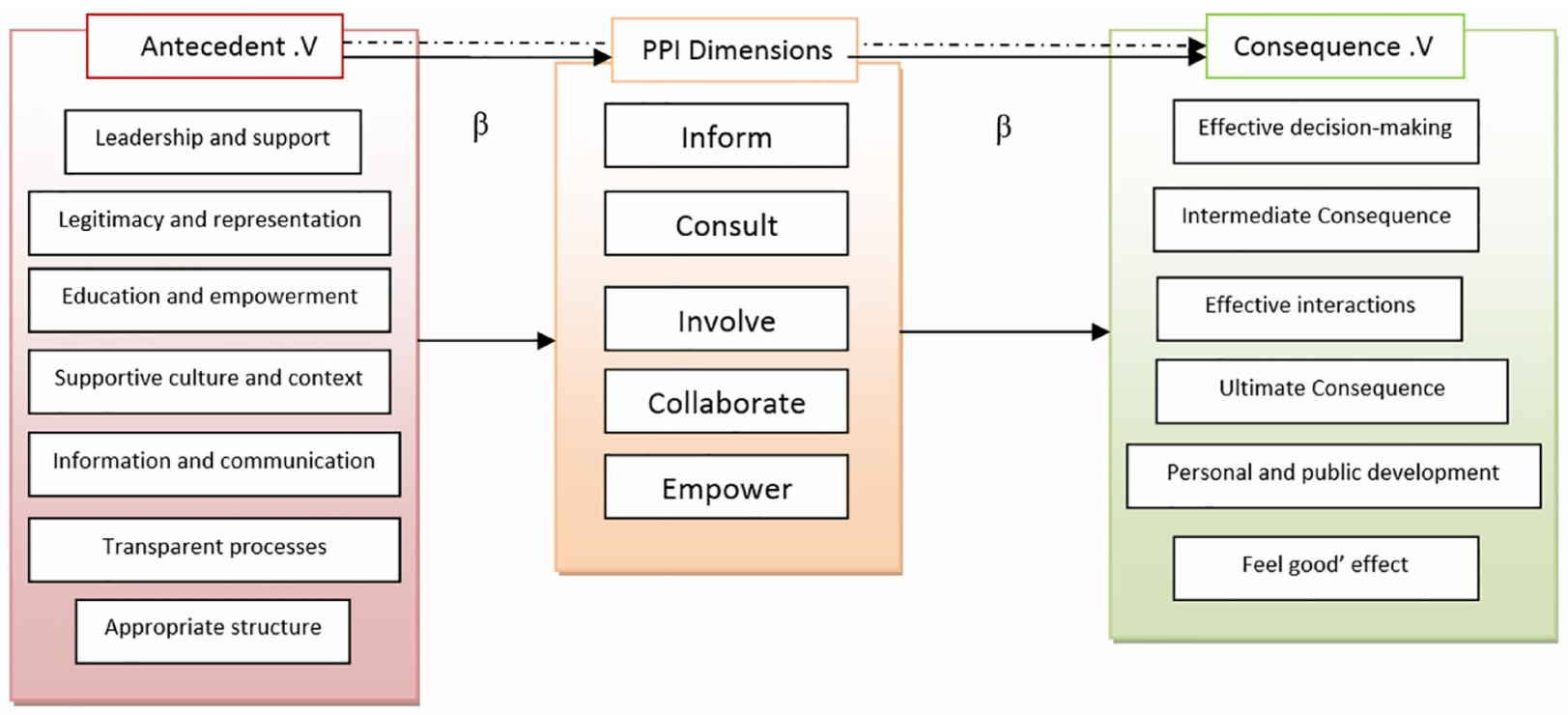

Figure 1. Proposed conceptual model of PPI in healthcare decision making

\section{Discussion}

Some variables were identified as antecedents, which was necessary to establish PPI in health care decision making. In this regard, "Leadership and support" emerged as a prerequisite for PPI initiatives. This factor also was emphasized by Carman (17), Boudioni (23), and Pivik (24). It seems that supportive frameworks, such as allocating financial resources, facilitating participation plans, and making mechanisms to encourage and create information technology infrastructure, as it was found in this study, can help to advance the goals of the PPI projects. Another important antecedent variables in this study were legitimacy and representation, which frequently are referred to in participation literature, as Sykes (25), Maloff (14), and Abelson (26) indicated. Having the legitimacy to represent the voices of patients and community participation is required. In this regard, representatives should be chosen so that they represent the community and are responsive to the community they serve. In this study, we found that education and empowerment are critical to the success of PPI. Marzano et al. (27) also emphasized the need for participants to have training and skills, as recommended by Kapiriri and colleagues (28), to equip people with the ability to make decisions. In this study, information and communication were considered to be the foundation for the PPI. Maloff (14) also emphasized that sufficient information is essential element for participation, and Tritter (29) encouraged people to apply dialogue for sharing problems and identify constraints and find meaningful solutions for problem which public encounter with them. Pivik et al. (24) emphacized citizens' participation in planning, and Maloff (14) also referred to consultation with the community. In this regard, Barnes et al. (30) emphasized designing a structure based on the democratic process, and Armstrong (31) suggested an informal, hierarchical structure for the successful implementation of the participation initiative. Many models of public involvement have been presented on a continuum. The most recognized of these is Arnstein's Eight Ladders of participation in which manipulation is located at the bottom, and citizen control at the top; this was the first model developed to explain involvement (40). Many adjustments have been created and evolved over time. Charles and DeMaio classified three degrees of control, ranging from 'consultation,' which is the lowest level of control, to "participation" and "manipulation" (41). The International Association for Public Participation (IAP2) has considered participation on a spectrum range from inform to empower and defined the goals and proposed methods to use for each level (42). In this study, we considered this continuum to show better the level of participation because of its transparency, simplicity, and completeness. According the findings, effective decision making has emerged as a consequence of the variables of PPI in healthcare decision making. Kolasa (35) believed that public participation could contribute to the transparency of the decision making process and Wait (38) indicated that cost-effective and safe decisions are the result of engaging people in the decision-making process. Another outcome of PPI is something we call 'intermediate consequence,' such as responsibility, responsiveness, democracy, justice, trust, and efficiency. These also were highlighted by some other authors (29, 30, 36-38. (Effective interactions also were an important results of PPI initiatives in our findings; Burns et al. (15) stated that, in participation schemes, patients could meet together, thereby creating improved relations and better communication between patients and staff members. We also found that the ultimate consequences were a favorable outcome of PPI. These outcomes ultimately are related to good 
health for citizens, which some authors have emphasized $(17,30,39)$. In this study, we also perceived that PPI can develop personal and public capacity. Burnes et al. (15) also declared that individual and community capacity building and skills development are the results of involving the public in decision making as well . Abelson (26) noticed that trained citizens to engage in participatory projects helps to achieve PPI goals. and finally feel good' effect, as Pizzo called it, and he believed that by implementing the participation of the public ensured that their needs would be expressed and thatb there would be some hope that they would be met (40). Some theoretical framework for public participation that provides a clear description of its components, specifies the public's participation techniques, the desired outcomes, and the proper environment is needed (41). Several public involvement models are available in the literature, and they try to show different aspects of community participation in the health system. The points that should be noted are 1) there is no comprehensive model that covers all aspects of involvement and 2) caution must be exercised in applying each model for a particular country or area (42). We should distinguish between the involvement model and methods because they are merely means of obtaining the views of the community or what is known as 'public input.' These models just focus on the level or intensity of participation on the basis of the amount of information exchanged, and they ignore the other aspects of the participation, such as leadership, organizational culture, necessary infrastructure to implement public involvement, and their consequences. So, in this model, we used a logical approach to provide a guide concerning how the public can become involved in the planning and decision making required in the healthcare system. In this regard, similar to each system that has components that include input, process, and outcome, we will provide a model that provides the necessary prerequisites and the potential consequences in compliance with the specific context of the Iranian healthcare system.

\section{Conclusions}

In this study, the main variables of PPI were identified by applying a logical approach. The antecedents, consequences, and dimensions of PPI were obtained from the literature and an in-depth qualitative assessment. Then, these variables were prioritized and weighted to develop a conceptual model. The identified variables present a framework to implement the PPI project in the Iranian healthcare system. This model can provide a practical guide for managers and policy makers to bring people to engage in decisions that influence their lives. Additional studies are needed to evaluate the application of PPI in other functions of the system, such as primary health care, developing guidelines, appraisals of health technology, and involving the public in healthcare research. To summarize, it has many social benefits to the community through involving an engaging patient and the public in decision-making and developing services.

\section{Acknowledgments:}

This is a study protocol of a Ph.D. thesis supported by Tehran University of Medical Sciences (TUMS) (Grant No.: TUMS-31393). The authors thank TUMS, which funded the research. We also appreciate all key informants who participated in this study.

\section{Conflict of Interest:}

There is no conflict of interest to be declared.

Authors' contributions:

All authors contributed to this project and article equally. All authors read and approved the final manuscript.

\section{References:}

1) Church J, Saunders D, Wanke M, Pong R, Spooner C, Dorgan M. Citizen Participation in health decisionmaking: past experience and future prospects. J Public Health Policy. 2002; 23(3): 12-32. doi: 10.2307/3343116, PMID: 12013713.

2) Declaration of Alma-Ata. International Conference on Primary Health Care, Alma- Ata, USSR, 6-12 September 1978. Available from: http://www.who.int/hpr/archive/docs/almaata.html

3) Department of Health. Patient and Public Involvement in the New NHS, London: Stationary Office, 1999.

4) Health Canada, Public Involvement: Model and Guidelines, Ottawa: Minister of Public Works and Government Services, 2000.

5) Kitzhaber JA, 1993. Prioritizing health services in an era of limits: the Oregon experience. B M J. 1993; 307(6900): 373-7. doi: 10.1136/bmj.307.6900.373, PMID: 8374424, PMCID: PMC1678226.

6) World Health Organization, Regional Health Systems Observatory- EMRO, Health Systems ProfileIslamic Republic of Iran, 2006. 
7) Aryankhesal A. The Iranian hospital grading system and its influence on stakeholders' behavior. $\mathrm{PhD}$ thesis. The University of York, 2010.

8) Rangrazjedi F, Rabiee R. Patient's Bill of Rights in Kashan`s hospitals (Iran). Behbood. 2005; 10(1): 62 71.

9) Joolaee S, Nikbakht-Nasrabadi, A, Parsa-Yekta, Z, Tschudin V, Mansouri I. An Iranian perspective on patients' rights. Nurs Ethics. 2006; 13(5): 488-502. PMID: 16961113.

10) Heyrani A, Maleki M, Marnani AB, Ravaghi H, Sedaghat M, Jabbari M, et al. Clinical governance implementation in a selected teaching emergency department: a systems approach. Implement Sci. 2012; 7: 84. PMID: 22963589.

11) Office of Hospital Management and Clinical Excellence: An introduction to essentials of clinical governance (in Persian). Tehran, Iran: Ministry of Health and Medical Education; 2011.

12) Ravaghi H, Mohseni M, Rafiei S, Zadeh NS, Mostofian F, Heidarpoor P. Clinical Governance in Iran: Theory to Practice. Procedia-Social and Behavioral Sciences. 2014; 109, 1174-9. doi: 10.1016/j.sbspro.2013.12.607.

13) Southampton University Hospitals NHS Trust (SUHT). Patient and Public Involvement Strategy 20112014. Available from: http://www.uhs.nhs.uk/

14) Maloff B, Bilan D, Thurston W. Enhancing public input into decision making: Development of the Calgary Regional Health Authority public participation model. Family \& Community Health. 2000; 23(1): 66-78. doi: 10.1097/00003727-200004000-00008

15) Burns KK, Bellows M, Eigenseher C, Gallivan J. Practical' resources to support patient and family engagement in healthcare decisions: a scoping review. BMC Health Serv Res. 2014; 14(1): 175. doi: 10.1186/1472-6963-14-175, PMID: 24735787, PMCID: PMC4005635.

16) Caddy J, Vergez C. Citizens as Partners: Information, Consultation and Public Participation in PolicyMaking. Paris: Organisation for Economic Cooperation and Development; 2001.

17) Carman KL, Dardess P, Maurer M, Sofaer S, Adams K, Bechtel C, Sweeney J. Patient and family engagement: a model for understanding the elements and developing interventions and policies. Health Affairs. 2013; 32(2): 223-31. doi: 10.1377/hlthaff.2012.1133.

18) Cleemput I, Christiaens W, Kohn L, Léonard C, Daue F, Denis A. Acceptability and Perceived Benefits and Risks of Public and Patient Involvement in Health Care Policy: A Delphi Survey in Belgian Stakeholders. Value in Health. 2015; 18(4): 477-83. doi: 10.1016/j.jval.2014.12.015.

19) Whitty JA, Burton P, Kendall E, Ratcliffe J, Wilson A, Littlejohns P, Scuffham PA. Harnessing the potential to quantify public preferences for healthcare priorities through citizens' juries. Int J Health Policy Manag. 2014; 3(2): 57-62. doi: 10.15171/ijhpm.2014.61, PMID: 25114943, PMCID: PMC4122074.

20) Christiaens W, Kohn L, Léonard C, Denis A, Daue F, Cleemput I. Models for citizen and patient involvement in health care policy Part I: exploration of their feasibility and acceptability. Health Services Research (HSR). Brussels: Belgian Health care Knowledge Centre (KCE). 2012. KCE Reports 195C. $\mathrm{D} / 2012 / 10.273 / 105$.

21) Morgan LM. Community participation in health: perpetual allure, persistent challenge. Health policy plan. 2001; 16(3): 221-30. doi: 10.1093/heapol/16.3.221, PMID: 11527862.

22) Momeni M. New topics in operations research, first edition, Tehran, Tehran University press, 2006

23) Boudioni M, McLaren S. Challenges and facilitators for patient and public involvement in England; focus groups with senior nurses. Open Journal of Nursing, 2013; 3(07): 472. doi: 10.4236/ojn.2013.37064.

24) Pivik JR. Practical strategies for facilitating meaningful citizen involvement in health planning. Commission on the Future of Health Care in Canada. 2002.

25) Sykes C, Goodwin W. Assessing patient, carer and public involvement in health care. Quality in Primary Care. 2007; 15(1): 45-52.

26) Abelson J, Forest PG, Eyles J, Smith P, Martin E, Gauvin FP, 2003. Deliberations about deliberative methods: issues in the design and evaluation of public participation processes. Social science \& medicine.2003; 57(2): 239-51. doi: 10.1016/S0277-9536(02)00343-X.

27) Marzano G, Lubkina V, Skurvydas A. Citizens' participation in healthcare: a productive opportunity or just a populist ethic idea? Hygeia. 2013; 48(2): 129-36.

28) Kapiriri L, Norheim OF, Heggenhougen K. Public participation in health planning and priority setting at the district level in Uganda. Health policy plan. 2003; 18(2): 205-13. PMID: 12740325.

29) Tritter JQ. Revolution or evolution: the challenges of conceptualizing patient and public involvement in a consumerist world. Health Expect. 2009; 12(3): 275-87. doi: 10.1111/j.1369-7625.2009.00564.x, PMID: 19754691. 
30) Barnes M, Coelho VS. Social participation in health in Brazil and England: inclusion, representation and authority. Health Expect. 2009; 12(3): 226-36. doi: 10.1111/j.1369-7625.2009.00563.x, PMID: 19754687.

31) Armstrong N, Herbert G, Aveling EL, Dixon Woods M, Martin G. Optimizing patient involvement in quality improvement. Health Expect. 2013; 16(3): e36-e47. doi: 10.1111/hex.12039.

32) Arnstein S. A ladder of citizen participation. American Institute of Planners Journal. 1969; 35: 216-24. doi: 10.1080/01944366908977225.

33) Charles CA, DeMaio S. Lay participation in health care decision-making: a conceptual model. J Health Polit Policy Law. 1993; 18: 881-904. doi: 10.1215/03616878-18-4-881.

34) International Association for Public Participation: IAP2 Spectrum of Public Participation

35) Kolasa K, Hermanowski T, Borek E. Importance of public participation in decision-making process in healthcare system illustarted with an example of the development of American and Polish scope of health benefit basket. Przegl Epidemiol. 2013; 67(4): 661-6, 749-54. PMID: 24741914.

36) Li KK, Abelson J, Giacomini M, Contandriopoulos D. Conceptualizing the use of public involvement in health policy decision-making. Soc Sci Med. 2015; 138: 14-21. doi: 10.1016/j.socscimed.2015.05.023, PMID: 26043073.

37) Linhorst DM, Eckert A, Hamilton G, Young E. The involvement of a consumer council in organizational decision making in a public psychiatric hospital. J Behav Health Serv Res. 2001; 28(4): 427-38. PMID: 11732245.

38) Wait S, Nolte E. Public involvement policies in health: exploring their conceptual basis. Health Econ Policy Law. 2006; 1(02): 149-62. doi: 10.1017/S174413310500112X.

39) Crawford MJ, Rutter D, Manley C, Weaver T, Bhui K, Fulop N, et al. Systematic review of involving patients in the planning and development of health care. BMJ. 2002; 325(7375): 1263. PMID: 12458240.

40) Pizzo E, Doyle C, Matthews R, Barlow J. Patient and public involvement: how much do we spend and what are the benefits? Health Expect. 2015; 18(6): 1918-26. doi: 10.1111/hex.12204, PMID: 12458240.

41) Thurston WE, MacKean G, Vollman A, Casebeer A, Weber M, Maloff B, et al. Public participation in regional health policy: a theoretical framework. Health Policy. 2005; 73(3): 237-52. doi: 10.1016/j.healthpol.2004.11.013, PMID: 16039343.

42) Ridley J, Jones L. User and public involvement in health services: A literature review. 2005; Partners in Change [Scottish Human Services Trust]. 